\title{
A Simplified Method For Economic Dispatch With Multiple Fuel Options
}

\author{
Ta Minh Cuong ${ }^{1}$ and Do Huynh Thanh Phong ${ }^{2}$ \\ ${ }^{1,2}$ Department of Electrical and Electronics Engineering, Ly Tu Trong college, Ho \\ Chi Minh city, Vietnam \\ No. 390,Hoang Van Thu street, Tan Binh district, Ho Chi Minh city, Vietnam \\ taminhcuong@gmai.com,dohuynhthanhphong@gmail.com
}

\begin{abstract}
The optimal generation dispatch problem with only one fuel option for each generating unit has been solved for many recent years. However, it is more realistic to represent the fuel cost function for each fossil fired plant as a segmented piece-wise quadratic functions. This is because of development of technology in thermal plants to reach maximum fuel save. Those units are faced with the difficulty of determining which the most economical fuel to burn is. This paper presents an approach to solve the unit commitment problem with multiple fuel options. An advantage of the method is to formulate Lagrange mathematical function easily based on the Lagrange multiplier theory. The proposed methods are tested on one test system consisting of ten generating units with various load demands and compared to other methods. The simulation results show that the methods are very efficient for the optimal generation dispatch problem with multiple fuel options.
\end{abstract}

Keywords: Economic load dispatch, multiple fuel options, Lagrange multiplier,

\section{Introduction}

In the power system planning and operation, the economic load dispatch (ELD) is meant the security of low-cost generating power as load demands according to the system constraints. This is the one of the most critical issues that has been researched recently. The single quadratic function for every generator unit prescribed basically cost function. In fact, piecewise quadratic functions is used to represent different fuels of each generator. These functions could be solved by Lagrange method.

Three approaches to cope with the simple ELD problem are the lambda-iteration method, base point with participation factors method and gradient method. Among these mentioned methods, the first one is the fastest and most effective method. These approaches assume that the cost function for individual generators is approximately the single quadratic function. The requirement of the above tactics is the increase of fuel cost curves which are piecewise linear and monotonically. The biggest advantage of these methods is simple to apply. However, in case the cost function has more than one variable, these techniques are failed. To overcome with challenge, the Newton method has been invented. This method is much closer to the minimum generation cost in one step than three above methods. Dynamic programming method (DP) is capable of dealing with non-monotonically and discontinuous incremental cost curves. Nonetheless, DP method was only accepted in the particular condition which add the ramp rate constrain of the units.

To surmount these confines of conventional methods, meta-heuristic approaches have been invented. These optimal techniques based on operational research and artificial intelligence concepts. Hopfield neural networks approach has been applied to solve the non-linear ELD problem, yet had two disadvantages. The first is the selection of appropriate weighting factors for the energy function. The second is the requirement of 
large computational burden to obtain an optimal solution. Simulated Annealing (SA) technique is similar to a local search technique. When this technique has been applied to a real system, setting of control parameter is a difficult task, and the convergence speed is slow. Genetic algorithm (GA) is the form of heuristic algorithm that mimics the process of natural evolution. This method has slow convergence near global optimum, sometimes may be trapped into local optimum. Evolutionary programming (EP) [1-3] is similar to genetic algorithm, tends to generate more effective and efficient than GA. However, both of GA and EP take long simulation time. Particle swarm optimization (PSO) is motivated by the simulation of social behavior of animal such as fish schooling and bird flocking. Although PSO can converge quickly towards the optimal solution, it has difficulties in reaching a global optimum and suffers from premature converge. Moreover, PSO has several control parameters. The convergence of the algorithm depends heavily on the value of its control parameters [4-5]. A novel optimization approach, Artificial Immune System (AIS) [6] has been applied the ELD problem. This approach utilizes the colonial selection principle and evolutionary approach wherein cloning of antibodies is performed followed by hyper mutation.

The convergence of these meta-heuristic may become the local optimum with long computational time when they deal with large-scale problems. The hybrid methods can overcome the main drawback from these methods. To utilize the advantages of the element methods integrated in the hybrid methods is aim of them. Some of these approaches in literature include Simulated Annealing - Particle Swarm Optimization (SAPSO) [7], Quantum-inspired version of the PSO using the harmonic oscillator (HQPSO) [8], Self-organizing hierarchical particle swarm optimization (SOH-PSO) [9], Bacterial foraging with Nelder-Mead algorithm (BF A-NM) [10], Adaptive Particle Swarm Optimization (APSO) [11], Uniform design with the genetic algorithm (UHGA) [12], Particle Swarm Optimization with chaotic and Gaussian approach (PSO-CG) [13], Self Tuning Hybrid Differential Evolution (STHDE) [14], variable Scaling Hybrid Differential Evolution (VSHDE) [15], Improved genetic algorithm with multiplier updating (IGAMU) [16], Differential evolution with sequential quadratic programming (DEC-SQP) [17], and Improved fast evolutionary programming (IEEP) [18]. Although the hybrid method can be better than the single approach, they can be slower.

In this paper, two effective methods based on the Lagrange multiplier theory are proposed in order to solve ELD problem. The advantage of the methods is that they are easy to formulate the problem mathematically from input data. An initial value of incremental cost is selected. In order to demonstrate the effectiveness of the proposed methods, they are tested on one test system having ten generating units with various load demands and compared to the methods such as Hierarchical Method (HM) [19], Hopfield Neural Network (HNN) [20], adaptive Hopfield neural network (AHNN) [21], , Improved Evolutionary Programming (IEP) [22], Modified Particle Swarm Optimization (MPSO) [23], Improved Fast Evolutioary Programming (IFEP) [24], Fast Evolutioary Programming (FEP) [24], Classical Evolutioary Programming (CEP) [24], and Enhanced Augmented Lagrange Hopfield Network method (EALHN) [25].

In this paper, an effective method based on the Lagrange multiplier theory is proposed in order to solve ELD problem. The effectiveness of the method is tested on one test system having ten generating units with various load demands.

\section{Problem Formulation}

Consider a power system consisting of $\mathrm{N}$ generating units, each loaded to $\mathrm{P}_{\mathrm{D}} \mathrm{MW}$. The generating units should be loaded in such a way that minimizes the total fuel $\operatorname{cost} \mathrm{C}_{\mathrm{T}}$ while satisfying the power balance and other constraints. In order to formulate the problem mathematically, the following notation is first introduced:
$C\left(P_{T m i}\right)$ :
Fuel cost of thermal plant, in $(R s / h)$. 
i: $\quad$ Index of generating units;

$k$ : Index of fuel types;

M: $\quad$ Number of fuel types of the generating unit get the most fuel types.

N: $\quad$ Number of online generating units;

$a_{i k}, b_{i k}, c_{i k}$ : Cost coefficients of ith generating unit with kth fuel type.

$P_{D}$ : Load demand of the system, in $M W$.

$\lambda$ : $\quad$ Lagrange multiplier.

$P_{L}$ : $\quad$ Total network loss of the system, in $M W$;

$P_{i k}: \quad \quad \quad$ Power of the ith generating unit with the kth fuel type, in $M W$;

$P_{i k}^{\min }:$ Minimum generation level of ith generating unit according to kth fuel type, in $M W$.

$P_{i k}^{\max }:$ Maximum generation level of ith generating unit according to kth fuel type, in $M W$.

The objective of the ED problem with multiple fuel options is only to minimize the total cost of thermal generating units while satisfying different constraints including power balance and generation limits.

$$
\operatorname{Min} C_{T}=\min \sum_{i=1}^{N} C_{T}\left(P_{i}\right)
$$

Where: $C_{T}\left(P_{i}\right)=\frac{1}{2} a_{i k} P_{i k}^{2}+b_{i k} P_{i k}+c_{i k}$

Subject to the following constraints:

1. Power balance constraint:

$$
\sum_{i=1}^{N} P_{i k}-P_{D}=0
$$

2)

2. The Constraints for each generating unit according to each fuel option:

$P_{i k}^{\min } \leq P_{i k} \leq P_{i k}^{\max }$ for fuel k

The Lagrange function $\mathrm{L}$ is formulated as follow:

$$
L=\sum_{i=1}^{N} C_{T}\left(P_{i k}\right)-\lambda\left(\sum_{i=1}^{N} P_{i k}-P_{D}\right)=0
$$

We have following equations by the partial derivatives of the Lagrange function with respect to according variables. Thus,

$\frac{\partial L}{\partial P_{i k}}=\frac{\partial C_{T}\left(P_{i k}\right)}{\partial P_{i k}}-\lambda=0$

From eq. (5), obtain following equations:

$$
\left.\begin{array}{c}
a_{1 k} P_{1 k}+b_{1 k}=a_{2 k} P_{2 k}+b_{2 k} \\
a_{1 k} P_{1 k}+b_{1 k}=a_{3 k} P_{3 k}+b_{3 k} \\
\vdots \\
a_{1 k} P_{1 k}+b_{1 k}=a_{N k} P_{N k}+b_{N k}
\end{array}\right\}
$$

Calculate generation of each unit using eqs. (6). 


$$
\left.\begin{array}{c}
P_{1 k}=\frac{a_{1 k}}{a_{1 k}} P_{1 k}+\frac{b_{1 k}-b_{1 k}}{a_{1 k}} \\
P_{2 k}=\frac{a_{1 k}}{a_{2 k}} P_{1 k}+\frac{b_{1 k}-b_{2 k}}{a_{2 k}} \\
\vdots \\
P_{N k}=\frac{a_{1 k}}{a_{N k}} P_{1 k}+\frac{b_{1 k}-b_{N k}}{a_{N k}}
\end{array}\right\}
$$

Obtain generation of each unit using eqs. (2) and (7)

(8)

$$
P_{1 k}=\frac{P_{D}-\sum_{i=1}^{N} B_{i k}}{\sum_{i=1}^{N} A_{i k}}
$$

$$
P_{i k}=A_{i k} P_{1 k}+B_{i k}
$$

Where:

$$
\left.\begin{array}{l}
\frac{a_{1 k}}{a_{i k}}=A_{i k} \\
\frac{b_{1 k}-b_{i k}}{a_{i k}}=B_{i k}
\end{array}\right\}
$$

\section{The Proposed Algorithm}

Figure 1 show a flowchart for solving the ELD problem using the proposed method.

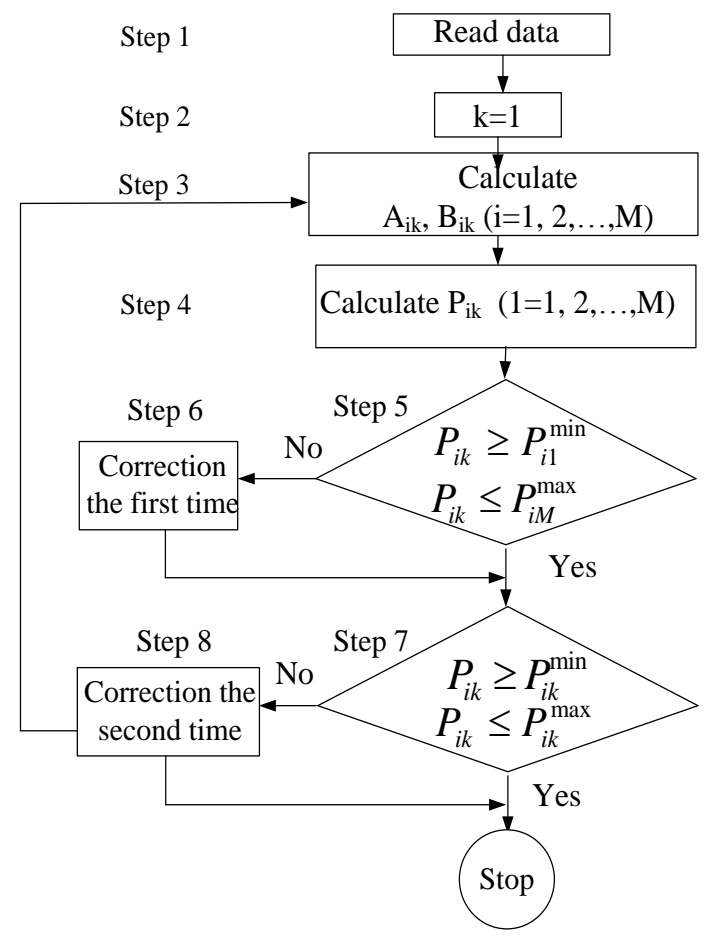

\section{Figure 1. The Flow Chart for Solving Economic Load Dispatch Problems}

Explanation for solving economic load dispatch problems:

1. Read data

2. Start with the first fuel type $(\mathrm{k}=1)$ for each generating unit.

3. Calculate $\mathrm{A}_{\mathrm{ik}}, \mathrm{B}_{\mathrm{ik}}$ using eqs (10). 
4. Calculate $P_{\mathrm{ik}}$ using eqs (8), (9).

5. Check $P_{i 1}^{\min } \leq P_{i k} \leq P_{i M}^{\max }$

If yes, go to step 7. Otherwise, go to step 6 .

6. If $P_{i k}<P_{i 1}^{\min }$, assign:

$\mathrm{a}_{\mathrm{ik}}=\mathrm{a}_{\mathrm{i} 1}, \mathrm{~b}_{\mathrm{ik}}=\mathrm{b}_{\mathrm{i} 1 ;} \mathrm{c}_{\mathrm{ik}}=\mathrm{c}_{\mathrm{i} 1}$

$P_{i k}=P_{i 1}^{\text {min }}$

If $P_{i k}>P_{i M}^{\max }$, assign:

$\mathrm{a}_{\mathrm{ik}}=\mathrm{a}_{\mathrm{iM}} ; \mathrm{b}_{\mathrm{ik}}=\mathrm{b}_{\mathrm{iM} ;} \mathrm{c}_{\mathrm{ik}}=\mathrm{c}_{\mathrm{iM}}$

$P_{i k}=P_{i M}^{\max }$

7. Check $P_{i k}^{\min } \leq P_{i k} \leq P_{i k}^{\max }$

If yes, go to step 9. Otherwise, go to step 8 .

8. If $P_{i k}<P_{i k}^{\min }$, assign:

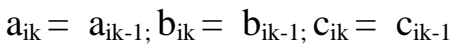

$P_{i k}^{\min }=P_{i k-1}^{\min } ; P_{i k}^{\max }=P_{i k-1}^{\max }$

If $P_{i k}>P_{i k}^{\max }$, assign:

$\mathrm{a}_{\mathrm{ik}}=\mathrm{a}_{\mathrm{ik}+1} ; \mathrm{b}_{\mathrm{ik}}=\mathrm{b}_{\mathrm{ik}+1}$; $\mathrm{c}_{\mathrm{ik}}=\mathrm{c}_{\mathrm{ik}+1}$

$P_{i k}^{\min }=P_{i k+1}^{\min } ; P_{i k}^{\max }=P_{i k+1}^{\max }$

9. Calculate generation cost

\section{Case Studies}

The proposed algorithm has been implemented in Mat lab 7.2 programming language and executed on Intel(R) Core (TM)2 Duo CPU T7250 @ 2.00GHZ (2CPU) laptop. The maximum difference between load demand and power generated from the set of available units is set to $10^{-4}$.

The test system consists of 10 generating units, each with two or three piecewise quadratic cost functions representing different fuel types. Total demands are gradually changed from 2,400 MW to 2,700 MW in steps of $100 \mathrm{MW}$ with power loss neglected. The results of the proposed approach are compared to those from [19-25] for various load demands of 2,400 MW, 2,500 MW, 2,600 MW, and 2,700 MW.

The comparisons for all load demand cases have revealed that the HM and HNN obtain the worst solution quality and HNN is the slowest algorithm for getting convergence. In fact, the HNN spends 60 seconds converging to optimal solution whereas other methods are just run around several seconds. In addition to obtaining high total fuel cost, the method violate the load balance constraints for al cases. HM in [19] tends to generate more power that that required from system whereas total power generated from HNN in [20] is less than system requirement. On the contrary, EALHN in [25] is the best technique as it gets good solution and very short computational time. In spite of the advantages, EALHN also suffers the difficult task for determining a set of control parameters. On the other hand, the EALHN may get local optimal solution if the best parameters during selection process are missed. For all cases, the proposed method shows its high performance due to high solution quality and short computational time obtained. The best solutions for the two methods are respectively indicated in Tables 5 . 
Table 1. Comparison of Fuel Cost and CPU Time for Load Demand of 2,400 MW

\begin{tabular}{|l|l|l|l|}
\hline Method & Total power & Cost (\$/h) & CPU time (s) \\
\hline HM [19] & $2,401.2$ & 488.50 & 1.08 \\
HNN [20] & $2,399.8$ & 487.87 & $\sim 60$ \\
AHNN [21] & 2,400 & 481.72 & $\sim 4$ \\
IEP [22] & 2,400 & 481.779 & - \\
MPSO [23] & 2,400 & 481.723 & - \\
EALHN [25] & 2,400 & 481.723 & 0.008 \\
Proposed method & $\mathbf{2 4 0 0 . 0}$ & $\mathbf{4 8 1 . 7 2 2 7}$ & $\mathbf{0 . 0 6 0}$ \\
\hline
\end{tabular}

Table 2. Comparison of Fuel Cost and CPU Time for Load Demand of 2,500 MW

\begin{tabular}{|l|l|l|l|}
\hline Method & Total power & Cost (\$/h) & CPU time (s) \\
\hline HM [19] & $2,500.1$ & 526.70 & - \\
HNN [20] & $2,499.8$ & 526.13 & $\sim 60$ \\
AHNN [21] & 2,500 & 526.230 & $\sim 4$ \\
IEP [22] & 2,500 & 526.304 & - \\
MPSO [23] & 2,500 & 526.239 & 6.1 \\
CEP [24] & 2,500 & 526.246 & 0.495 \\
FEP [24] & 2,500 & 526.262 & 0.394 \\
IFEP [24] & 2,500 & 526.246 & 0.558 \\
EALHN [25] & 2,500 & 526.239 & 0.006 \\
Proposed method & $\mathbf{2 5 0 0}$ & $\mathbf{5 2 6 . 2 3 9}$ & $\mathbf{0 . 0 5 8}$ \\
\hline
\end{tabular}

Table 3. Comparison of Fuel Cost and CPU time for Load Demand of 2,600 MW

\begin{tabular}{|l|l|l|l|}
\hline Method & Total power & Cost (\$/h) & CPU time (s) \\
\hline HM [19] & $2,599.3$ & 574.03 & - \\
HNN [20] & $2,599.8$ & 574.26 & $\sim 60$ \\
AHNN [21] & 2,600 & 574.37 & $\sim 4$ \\
IEP [22] & 2,600 & 574.473 & - \\
MPSO [23] & 2,600 & 574.381 & - \\
EALHN [25] & 2,600 & 574.381 & 0.005 \\
Proposed method & $\mathbf{2 6 0 0}$ & $\mathbf{5 7 4 . 3 8 0}$ & $\mathbf{0 . 0 5 8}$ \\
\hline
\end{tabular}

Table 4. Comparison of Fuel Cost and CPU Time for Load Demand of 2,700 MW

\begin{tabular}{|l|l|l|l|}
\hline Method & Total power & Cost $\mathbf{( \$ / h )}$ & CPU time (s) \\
\hline HM [19] & $2,702.2$ & 625.18 & - \\
HNN [20] & $2,699.7$ & 626.12 & $\sim 60$ \\
AHNN [21] & 2,700 & 626.24 & $\sim 4$ \\
IEP [22] & 2,700 & 623.851 & - \\
MPSO [23] & 2,700 & 623.809 & - \\
EALHN [25] & 2,700 & 623.809 & 0.013 \\
Proposed method 2 & $\mathbf{2 7 0 0}$ & $\mathbf{6 2 3 . 8 0 9 2}$ & $\mathbf{0 . 0 5}$ \\
\hline
\end{tabular}


Table 5. Optimal Solution Obtained by Proposed Method

\begin{tabular}{|l|l|l|l|l|l|l|l|l|}
\hline \multirow{2}{*}{ Unit } & \multicolumn{2}{|l|}{$\mathbf{P}_{\mathbf{D}}=\mathbf{2 4 0 0} \mathbf{M W}$} & \multicolumn{2}{l|}{$\mathbf{P}_{\mathbf{D}}=\mathbf{2 5 0 0} \mathbf{M W}$} & \multicolumn{2}{l|}{$\mathbf{P}_{\mathbf{D}}=\mathbf{2 6 0 0} \mathbf{M W}$} & \multicolumn{2}{|l|}{$\mathbf{P}_{\mathbf{D}}=2700 \mathbf{M W}$} \\
\cline { 2 - 10 } & $\begin{array}{l}\text { Fuel } \\
\text { type }\end{array}$ & $\mathbf{G e n}$ & $\begin{array}{l}\text { Fuel } \\
\text { type }\end{array}$ & & $\begin{array}{l}\text { Fuen } \\
\text { type }\end{array}$ & Gen & $\begin{array}{l}\text { Fuel } \\
\text { type }\end{array}$ & Gen \\
\hline 1 & 1 & 189.7405 & 2 & 206.5190 & 2 & 216.5442 & 2 & 218.2527 \\
\hline 2 & 1 & 202.3427 & 1 & 206.4573 & 1 & 210.9058 & 1 & 211.6691 \\
\hline 3 & 1 & 253.8953 & 1 & 265.7391 & 1 & 278.5441 & 1 & 280.6653 \\
\hline 4 & 3 & 233.0456 & 3 & 235.9531 & 3 & 239.0967 & 3 & 239.6167 \\
\hline 5 & 1 & 241.8297 & 1 & 258.0177 & 1 & 275.5194 & 1 & 278.5361 \\
\hline 6 & 3 & 233.0456 & 3 & 235.9531 & 3 & 239.0967 & 3 & 239.6299 \\
\hline 7 & 1 & 253.2750 & 1 & 268.8635 & 1 & 285.7170 & 1 & 288.6616 \\
\hline 8 & 3 & 233.0456 & 3 & 235.9531 & 3 & 239.0967 & 3 & 239.6352 \\
\hline 9 & 1 & 320.3832 & 1 & 331.4877 & 1 & 343.4934 & 3 & 428.49 \\
\hline 10 & 1 & 239.3969 & 1 & 255.0562 & 1 & 271.9861 & 1 & 274.8433 \\
\hline $\begin{array}{l}\text { Total } \\
\text { power } \\
\text { (MW) }\end{array}$ & $\mathbf{2 4 0 0}$ & & & & & & & \\
\hline
\end{tabular}

\section{Conclusions}

A simplified methodology has been developed in this paper to solve the problem of economic load dispatch with multiple fuel options in which the generator cost functions are represented as piece-wise quadratic cost functions. The test results for the example problems bring out the advantages of the proposed method. This makes the proposed method computationally faster. The convergence abilities of the method are better than almost methods. The simulation results of fuel cost and time to reach convergence show that it can be applied to a wide range of optimization problems.

\section{References}

[1] H. T. Yang, P. C. Yang and C. L. Huang, "Evolutionary programming based economic dispatch for units with non-smooth fuel cost function", IEEE Transaction on Power Systems, vol. 11, no. 1, (1996), pp. $112-118$.

[2] N. Sinha, R. Chakrabarti and P. K. Chattopadhyay, "Evolutionary programming techniques for economic load dispatch", IEEE Trans. on Evolutionary Computation, vol. 7, no. 1, (2003), pp. 83-94.

[3] T. Jayabarathi, K. Jayaprakash, D. N. Jayakumar and T. Raghunathan, "Evolution programing techniques for different kinds of economic dispatch problems", Elect. Power Syst. Res., vol. 73, (2008), pp. 1322-1331.

[4] Z.-L. Gaing, "Paricle swarm optimization to solving the economic dispatch", IEEE Trans Power Syst, vol. 18 , no. 3, (2003), pp. 1187-1195.

[5] T. A. A. Victoire and A. E. Jeyakumar, "Discussion of particle swarm optimization to solving the economic dispatch considering the generator constraints", IEEE Trans. on Power Systems, vol. 19, no. 4, (2004), pp. 2121-2123.

[6] K. Panigrahi, S. R. Yadav and S. Agrawal, "A clonal algorithms to solve economic load dispatch", Elect. Power Syst. Res., vol. 77, no. 10, (2007), pp. 1381-1389.

[7] C. C. Kuo, "A novel coding schemefor practical economic dispatch by", IEEE Trans. Power Syst., vol. 23, no. 4, (2008), pp. 1825-1835.

[8] L. D. S. Mariani and V. C. Coelho, "Particle swarm approach based on quantum mechanics and harmonic oscillator potential well for economic load dispatch with valve-point effects", Energy Convers. Manage, vol. 49, no. 11, (2008), pp. 3080-3085.

[9] K. T. Chaturvedi, M. Pandit and L. Srivastava, "Self-organizing hierarchical particle swarm optimization for nonconvex economic dispatch", IEEE Trans. Power Syst, vol. 23, no. 3, (2008), pp. 1079-1087.

[10] B. K. Pandi and V. R.Panigrahi, "Bacterial foraging optimisation: Nelder-Mead hybrid algorithm for economic load dispatch”, IET Gen., Transm., Distrib., vol. 2, no. 4, (2008), pp. 556-565.

[11] K. Panigrahi, V. R. Pandi and S. Das, "Adaptive particle swarm optimization approach for static and dynamic economic load dispatch”, Energy Convers. Manage, vol. 49, no. 6, (2008), pp. 1407-1415. 
[12] D. K. He, F. L. Wang and Z. Z. Mao, "Hybrid genetic algorithm for economic dispatch with valve-point effect", Elect. Power Syst. Res., vol. 78, no. 4, (2008), pp. 626-633.

[13] L. D. S. Lee and C. S. Coelho, "Solving economic load dispatch problems in power systems using chaotic and Gaussian particle swarm optimization approaches", Int. Journal of Elect. Power Energy Syst., vol. 30, no. 4, (2008), pp. 297-307.

[14] S. K. Wang, J. P. Chiou and C. W. Liu, "Non-smooth/non-convex economic dispatch by a novel hybrid differential evolution algorithm", IET Gen., Transm., Distrib., vol. 1, no. 5, (2007), pp. 793-803.

[15] J. P. Chiou, "Variable scaling hybrid differential evolution for large scale economic dispatch problems", Elect. Power Syst. Res., vol. 77, no. 1, (2007), pp. 212-218.

[16] C. L. Chiang, "Genetic-based algorithm for power economic load dispatch", IET Gen., Transm., Distrib., vol. 1, no. 2, (2007), pp. 261-269.

[17] L. D. S. Mariani and V. C. Coelho, "Correction to combine of chaotic differential evolution and quadratic programming for economic dispatch optimization with valve-point effect", IEEE Trans. Power Syst., vol. 21, no. 3, (2006), pp. 1465-1465.

[18] N. Sinha, R. Chakrabarti and P. K. Chattopadhyay, "Evolutionary programming techniques for economic load dispatch", IEEE Trans. Evol., vol. 7, no. 1, (2003), pp. 83-94.

[19] C. E. Lin and G. L. Viviani, "Hierarchical economic dispatch for piecewise quadratic cost function", IEEE Transactions on Power Systems, vol. PAS-103, no. 6, (1984), pp. 1170-1175.

[20] J. H. Park, Y. S. Kim, I. K. Eom and K. Y. Lee, "Economic load dispatch for piecewise quadratic cost function using Hopfield Neural Network", IEEE Transactions on Power Systems, vol. 8, no. 3, (1993), pp. 1030-1038.

[21] K. Y. Lee, A. Sode-Yome and J. H. Park, "Adapive Hopfield Neural Network for economic load dispatch", IEEE Transactions on Power Systems, vol. 13, no. 13, (1998), pp. 519-526.

[22] Y. M. Park, J. R. Won and J. B. Park, "A new approach to economic load dispatch based on Improved Evolutionary Programming", Engineering Intelligent Systems Electrical Engineering Communication, vol. $6,(\mathbf{1 9 9 8})$, pp. 103-110

[23] J. B. Park, K. S. Lee, J. R. Shin and K. Y. Lee, "A particle swarm opimization for economic dispatch wih nonsmooth cost functions", IEEE Transactions on Power Systems, vol. 20, no. 1, (2005), pp. 34-42.

[24] T. Jayabarathi, K, Jayaprakash, D. N. Jayakumar and T. Raghunathan, "Evolutionary Programming techniques for different kinds of economic dispatch problems", Electric PowerSystems Reasearch, vol. 73, no. 2, (2005), pp. 169-176.

[25] V. N. D. Ongsakul, "Economic dispatch with multiple fuel types by Enhanced Augmented Lagrange Hopfield Network”, Power System Technology and IEEE Power India Conference, (2008).
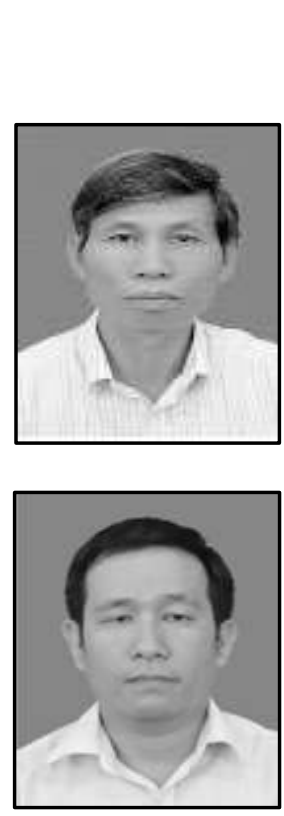

\section{Authors}

Ta Minh Cuong, He received his B.Eng. in Electrical Engineering from University of Technical education Ho Chi Minh City, Ho Chi Minh city, Vietnam in 1999. He received his M.Eng. in Electrical Engineering from University of Technical Ho Chi Minh City, Ho Chi Minh city, Vietnam in 2012. Now, he is teaching at department of electrical and electronics engineering, Ly Tu Trong college, Ho Chi Minh city, Vietnam.

Do Huynh Thanh Phong, He received his B.Eng. and M.Eng degrees in Electrical Engineering from University of Technical education Ho Chi Minh City, Ho Chi Minh city, Vietnam in 2008 and 2013, respectively. He is currently teaching at Faculty of Electrical and Electronics Engineering, Ly Tu Trong Technical College, HCM City, Vietnam. His research interests are optimal power flow and improvement of electricity quality. 